\title{
On quarks and the origin of QCD \\ Partons and baryons from intrinsic states
}

Trinhammer, Ole L.

Published in:

Epl

Link to article, DOI:

10.1209/0295-5075/133/31001

Publication date:

2021

\section{Document Version}

Publisher's PDF, also known as Version of record

Link back to DTU Orbit

Citation (APA):

Trinhammer, O. L. (2021). On quarks and the origin of QCD: Partons and baryons from intrinsic states. Epl, 133(3), [31001]. https://doi.org/10.1209/0295-5075/133/31001

\section{General rights}

Copyright and moral rights for the publications made accessible in the public portal are retained by the authors and/or other copyright owners and it is a condition of accessing publications that users recognise and abide by the legal requirements associated with these rights.

- Users may download and print one copy of any publication from the public portal for the purpose of private study or research.

- You may not further distribute the material or use it for any profit-making activity or commercial gain

- You may freely distribute the URL identifying the publication in the public portal

If you believe that this document breaches copyright please contact us providing details, and we will remove access to the work immediately and investigate your claim. 
OPEN ACCESS • OPEN ACCESS

On quarks and the origin of QCD: Partons and baryons from intrinsic states

To cite this article: Ole L. Trinhammer 2021 EPL 13331001

View the article online for updates and enhancements.
You may also like

- Topological Objects in Holographic QCD
Hideo Suganuma and Keiichiro Hori
- The chiral model of Sakai-Sugimoto at
$\frac{\text { finite baryon density }}{\text { Keun-Young Kim, Sang-Jin Sin and Ismail }}$
Zahed
- A review of the open charm and open
$\frac{\text { bottom systems }}{\text { Hua-Xing Chen, Wei Chen, Xiang Liu et al. }}$




\title{
On quarks and the origin of QCD: Partons and baryons from intrinsic states
}

\author{
Ole L. TRinhammer
}

Department of Physics, Technical University of Denmark - Fysikvej bld. 30\%, DK 2800 Kongens Lyngby, Denmark

received 7 October 2020; accepted in final form 5 January 2021 published online 30 March 2021

PACS 12.40.Yx - Hadron mass models and calculations PACS 12.90.+b-Miscellaneous theoretical ideas and models

\begin{abstract}
We create quarks from baryons in stead of constituting baryons from quarks. The quantum fields of QCD are generated via the exterior derivative (momentum form) of baryon wave functions on an intrinsic configuration space, the Lie group $U(3)$. Local gauge transformations correspond to coordinate translations in the intrinsic space. A proton spin structure function and a proton magnetic moment are derived. We show how the spectrum of unflavoured baryons, the $N$ and Delta resonances, can be understood from a mass Hamiltonian on the intrinsic space and note how our model resolves the problem of colour confinement. We calculate an approximate value for the relative neutron-to-proton mass shift and give an exact value for the neutron mass. We predict neutral charge singlets that may be interpreted as neutral pentaquarks at $\mathrm{LHCb}$.
\end{abstract}

openlaccess Copyright (C) 2021 The author(s)

Published by the EPLA under the terms of the Creative Commons Attribution 4.0 International License (CC BY). Further distribution of this work must maintain attribution to the author(s) and the published article's title, journal citation, and DOI.

Introduction. - Nature, at the present level of our understanding, exhibits degrees of freedom that we call quarks. Quarks are described as fractionally charged spinone-half particles of different kinds, labelled by flavour and colour. The constituent quark model for baryons like the proton and the neutron carries with it for many years a missing resonance problem [1,2]: many more resonances are predicted than observed ${ }^{1}$. Quantum chromodynamics [1] carries with it a confinement problem: colour degrees of freedom are confined. Confinement has not yet been shown to follow from the QCD Lagrangian density. In the present work we try to solve these two seemingly independent problems by a common idea: we consider colour to live in a compact, intrinsic space, the Lie group $U(3)$ -much like a generalized spin variable with QCD as a projection. Compactness confines colour per construction.

In 1925 George Uhlenbeck and Samuel Goudsmit [4] realised that the intrinsic angular momentum, the spin, of

\footnotetext{
${ }^{1}$ The constituent quark model was successful in predicting, e.g., the Omega minus baryon at $1685 \mathrm{MeV}$ [3]. Today however, the multiplet idea is mostly used post festum to label resonances when they are already discovered. The present model uses a compact configuration space which gives a periodic potential that lifts higher lying levels out of the "resonance domain".
}

the electron represents a new, intrinsic, degree of freedom. We generalise this insight to cover also colour, flavour and electric charge. This is possible by combining the strong and electroweak interactions into a description of baryons as stationary states on an intrinsic configuration space, the Lie group $U(3)$. This space contains all three gauge groups of the standard model as subspaces which is a first motivation to choose it as a configuration space for baryons. It is compact and has nine generators equivalent to the nine kinematic generators in the laboratory space: momentum, angular momentum and Laplace-Runge-Lenz generators [5] which could explain its origin. Baryons feel both the strong interactions with $S U(3)$ symmetry and electroweak interactions with $U(1) \times S U(2)$ symmetry. We show that coordinate translations in the intrinsic space equate local gauge transformations in the laboratory space. We also show that the fundamental fields of quantum chromodynamics, quarks and gluons, are generated by the exterior derivative, the momentum form, on the intrinsic wave function. To give an intuitive picture, imagine playing "ducks and drakes" where a stone thrown at a small glancing angle scatters on a water surface and creates ripples on the surface where it hits. The 
ripple patterns are quantised to fit the compact intrinsic space for mass eigenstates. The three Abelian momentum generators excite toroidal orbits which we interpret as colour degrees of freedom. The off-toroidal generators excite non-commuting degrees of freedom taking care of spin and flavour via off-toroidal derivatives in the Laplacian on $U(3)$.

The quarks in the present model are not fundamental [6] but share gauge groups with standard model quarks. Quarks are intrinsic orbits in the baryon excited in scattering by flavour generators which use quantum numbers for quark charge and baryon hypercharge as coefficients on their colour generators. The quark masses may be related to the curvature of such orbits when embedded in laboratory space, see footnote ${ }^{3}$.

The creation of unit electric charge, e.g., in the $n \rightarrow p$ decay is interpreted topologically to originate in period doublings in the intrinsic nucleon wave function. The decay relates the strong and electroweak sectors to yield equations for the electroweak energy scale and the Higgs mass in closed forms [7] and relates the electroweak mixing angle $\theta_{W}$ and the Cabibbo angle $\theta_{C}$ via quark flavour generators $T_{u}, T_{d}$ and $T_{s}$ to have $\sin ^{2} \theta_{W} \approx 1-\operatorname{Tr} T_{u}^{\dagger} T_{d}=$ $2 / 9,\left|\sin \theta_{C}\right| \approx\left|\operatorname{Tr} T_{u}^{\dagger} T_{s}\right|=2 / 9$ [8]. The $u, d$ flavour generators also enter our treatment of the electroweak sector via a slight change in the electroweak energy scale $v=$ $v_{\mathrm{SM}} / \sqrt{\left|V_{u d}\right|}$. It may be a matter of definition whether this should be ascribed to a change in the electroweak interactions of quarks. We prefer to see it as a shift in interpretation by redefining electroweak coupling contants [8] even though signal strengths $\mu_{H V V} / \mu_{H V V, \mathrm{SM}}=\frac{1}{\left|V_{u d}\right|}=1.03$ will distinguish the viewpoints [7]. In the present work, however, we focus our attention on the strong interactions.

Baryons as intrinsic configuration states. Baryons are considered as stationary states on an intrinsic configuration space, the Lie group $U(3)$. We identify baryon masses as the energy eigenvalues of [9]

$$
\frac{\hbar c}{a}\left[-\frac{1}{2} \Delta+\frac{1}{2} d^{2}(e, u)\right] \Psi(u)=\mathcal{E}_{0} \Psi(u), \quad u \in U(3),
$$

with $m c^{2}=\mathcal{E}_{0}$. The length scale $a$ is related to the classical electron radius $r_{\mathrm{e}}=\frac{e^{2}}{4 \pi \epsilon_{0} m_{\mathrm{e}} c^{2}}[10,11]$ by the expression $r_{\mathrm{e}}=\pi a$ [9] from mapping of the intrinsic baryon dynamics to the laboratory space. The factor $\pi$ reflects the toroidal shape of the intrinsic configuration space $U(3)$. The baryonic energy scale $\Lambda=\hbar c / a=\frac{\pi}{\alpha} m_{\mathrm{e}} c^{2}$ is close to the scale of non-pertubative quantum chromodynam$\operatorname{ics} \Lambda \frac{(5)}{\mathrm{MS}} \approx 210(14) \mathrm{MeV}$ [12]. The Hamiltonian in (1) is radically reinterpreted from lattice gauge theory [13] with a potential inspired by [14]. The unitary configuration variable $u \in U(3)$ now concerns the full baryon configuration and has eigenvalues $e^{i \theta_{j}}, j=1,2,3$ given by three dynamical eigenangles $\theta_{j} \in \mathbb{R}$ which we interpret as colour degrees of freedom, confined per construction by the compactness of $U(3)$. These eigenangles can be used for a polar decomposition of the Laplacian [15]

$$
\Delta=\sum_{j=1}^{3} \frac{1}{J^{2}} \frac{\partial}{\partial \theta_{j}} J^{2} \frac{\partial}{\partial \theta_{j}}-\sum_{\substack{1 \leq i<j \leq 3 \\ k \neq i, j}}^{3} \frac{\left(S_{k}^{2}+M_{k}^{2}\right) / \hbar^{2}}{8 \sin ^{2} \frac{1}{2}\left(\theta_{i}-\theta_{j}\right)}
$$

where [16]

$$
J=\prod_{1 \leq i<j \leq 3}^{3} 2 \sin \frac{1}{2}\left(\theta_{i}-\theta_{j}\right)
$$

and the generators $S_{k}$ and $M_{k}$ take care of spin and flavour, respectively. The potential in (1) is constructed from the shortest geodesic $d(e, u)$ from the neutral element $e$, the origo in the configuration space. The potential is periodic in the eigenangles and depends only on these [17]

$$
\frac{1}{2} d^{2}(e, u)=\frac{1}{2} \operatorname{Tr} \chi^{2}=\sum_{j=1}^{3} w\left(\theta_{j}\right), u=e^{i \chi}
$$

where the generator

$\chi=\theta_{j} T_{j}+\left(\alpha_{j} S_{j}+\beta_{j} M_{j}\right) / \hbar, i T_{j}=\frac{\partial}{\partial \theta_{j}} ; \quad \alpha_{j}, \beta_{j} \in \mathbb{R}$

and (see fig. 1)

$w(\theta)=\frac{1}{2}(\theta-n \cdot 2 \pi)^{2}, \quad \theta \in[(2 n-1) \pi,(2 n+1) \pi], \quad n \in \mathbb{Z}$.

The periodicity of the potential reflects the compactness of the configuration space. We see that (1) with (2) inserted is analogous to solving the hydrogen atom [18]. Now however, there are three "radial" degrees of freedom, the three eigenangles $\theta_{j}$ that span the Abelian (maximal) torus (7) of $U(3)$. The independence of the potential upon the remaining six dynamical variables $\alpha_{j}, \beta_{j}$ follows from the invariance of the trace on similarity operations $u \rightarrow v^{-1} u v, v \in U(3)$, in particular the ones that diagonalise $u$, thus

$$
u \sim v^{-1} u v=\left(\begin{array}{lll}
e^{i \theta_{1}} & & \\
& e^{i \theta_{2}} & \\
& & e^{i \theta_{3}}
\end{array}\right) .
$$

The length scale $a$ in (1) can be used to map from the laboratory space to the intrinsic space by the identification, see fig. 1,

$$
\theta_{j}=x_{j} / a
$$

We imagine the mapping in (8) to take place in scattering experiments where the intrinsic degrees of freedom are excited by the nine kinematic generators from laboratory space, namely momentum

$$
p_{j}=\frac{-i \hbar}{a} \frac{\partial}{\partial \theta_{j}}=\frac{\hbar}{a} T_{j},
$$

angular momentum, e.g.,

$$
S_{1}=a \theta_{2} p_{3}-a \theta_{3} p_{2}=\hbar \lambda_{7},
$$




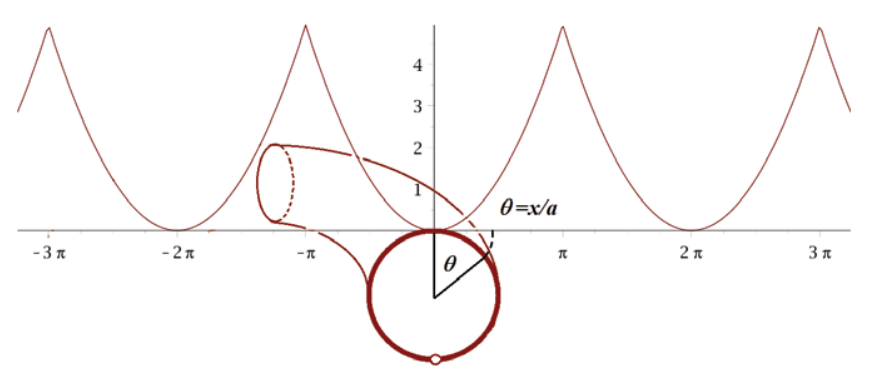

Fig. 1: Trace potential (6) in dynamical variables $\theta$ and mapping into toroidal eigenangles $\theta_{j}=x_{j} / a, j=1,2,3$ from laboratory space. The length scale $a$ is taken from the classical electron radius $r_{e}[10,11]$ as $\pi a=r_{e}[9]$. Figure elaborated from [19].

and Laplace-Runge-Lenz operators, e.g.,

$$
M_{1} / \hbar=\theta_{2} \theta_{3}+\frac{a^{2}}{\hbar^{2}} p_{2} p_{3}=\lambda_{6}
$$

Here, the lambdas are the Gell-Mann generators [5].

The quantisation inherent in (9) generalises to all of the configuration space by the global concepts of left (or right) invariant coordinate fields $\partial_{j}$ and corresponding coordinate forms $\mathrm{d} \theta_{j}[20]$

$$
\begin{aligned}
\partial_{j} & \left.\equiv \frac{\partial}{\partial \theta} u e^{\theta i T_{j}}\right|_{\theta=0}=u i T_{j}, \\
{\left[\theta_{i},\left.\frac{\partial}{\partial \theta_{j}}\right|_{e}\right] } & =\delta_{i j} \rightarrow \mathrm{d} \theta_{i}\left(\partial_{j}\right)=\delta_{i j} .
\end{aligned}
$$

This generalises the well-known commutation relations

$$
\left[a \theta_{i}, p_{j}\right]=-i \hbar \delta_{i j}
$$

From the coordinate representations [5] in (10) and (11)

$$
\left[M_{i}, M_{j}\right]=\left[S_{i}, S_{j}\right]=-i \hbar \varepsilon_{i j k} S_{k}
$$

Note the minus sign for the spin commutators as in body fixed intrinsic coordinate systems in nuclear physics [21].

Quarks and gluons as scattering states. - In solving (1) we introduce the measure-scaled wave function [9]

$$
\Phi(u) \equiv J \Psi(u) \equiv R\left(\theta_{1}, \theta_{2}, \theta_{3}\right) \Upsilon\left(\alpha_{1}, \alpha_{2}, \alpha_{3}, \beta_{1}, \beta_{2}, \beta_{3}\right) .
$$

The two functions $R$ and $\Upsilon$ are analogues of the radial wave function and the spherical harmonics used in describing the Euclidean three-dimensional case of the hydrogen atom [18].

We consider quarks and gluons as scattering states on baryons. We create their corresponding fields $\psi_{j}$ and $G^{(k)}$ via the momentum forms $\mathrm{d} R$ and $\mathrm{d} \Phi$ acting on the toroidal generators $i T_{j}$ and the basis $i \lambda_{k}$ of the adjoint representation of the $s u(3)$ algebra, respectively,

$$
\psi_{j}(u)=\mathrm{d} R_{u}\left(\partial_{j}\right), \quad G^{(k)}(u)=\mathrm{d} \Phi_{u}\left(\partial_{k}\right) .
$$

Here

$$
\partial_{k}=\text { uit }_{k}, \quad k=1,2, \ldots 8
$$

are left invariant coordinate fields corresponding to the gluon field generators $t_{k}=\lambda_{k} / 2$ [1], six of which are proportional to $S_{j}$ and $M_{j}$ and the remaining two are diagonal linear combinations of the $T_{j}$ 's. The momentum form becomes "operational" by derivation of $\Phi$ along the direction given by the generator. For a generator $Z$ we have the general definition of a derivation as

$$
Z[\Phi](u) \equiv \mathrm{d} \Phi_{u}(Z)=\left.\frac{\mathrm{d}}{\mathrm{d} t} \Phi\left(u e^{t Z}\right)\right|_{t=0}, \quad Z \in u(3),
$$

where $u(3)$ is the algebra of $U(3)$.

From (16) applied to the ground state of (1) we have derived exemplar parton distribution functions for the valence $u$ and $d$ quarks of the proton [9] and we have recently derived exemplar energy-momentum tensor components of the proton too [22]. We add here as a further motivation a proton spin structure function and the proton magnetic moment. For an approximate calculation we use a Slater determinant $R$ with period doubling in the eigenangles interpreted as a topological origin of the proton's electric charge [9]

$$
R=\frac{1}{N}\left|\begin{array}{ccc}
1 & 1 & 1 \\
\sin \frac{1}{2} \theta_{1} & \sin \frac{1}{2} \theta_{2} & \sin \frac{1}{2} \theta_{3} \\
\cos \theta_{1} & \cos \theta_{2} & \cos \theta_{3}
\end{array}\right| .
$$

Here $N$ with $N^{2}=\frac{3}{2} \pi^{3}-\frac{44}{3} \pi$ normalises $R$ on $\theta_{j} \in[0, \pi]$. The quark distribution functions derived in [9] are obtained along one-parameter curves generated by the momentum form $\mathrm{d} R$ applied to flavour generators $T_{q}$ (23) while summing over colours and squaring to get probability distributions for the parton momentum fraction $x \in[0,1]$

$$
f_{q}(x) \mathrm{d} x=\left(\sum_{j=1}^{3} \mathrm{~d} R_{u=\exp \left(\theta i T_{q}\right)}\left(i T_{j}\right)\right)^{2} \mathrm{~d} \theta .
$$

Here $\theta=\pi \xi$ and the boost variable $\xi$ is [9]

$$
\xi \equiv \frac{\mathcal{E}-\mathcal{E}_{0}}{\mathcal{E}}=\frac{2-2 x}{2-x}, \quad \mathcal{E}_{0}=m c^{2},
$$

from impacting a massless energy momentum $q=(\mathcal{E}-$ $\left.\mathcal{E}_{0}, \mathbf{q}\right)$ on a parton $x P$ acquiring mass $x \mathcal{E}$ in a proton at rest $\left(\mathcal{E}_{0}, \mathbf{0}\right)$, i.e.,

$$
\left(x P_{\mu}+q_{\mu}\right)\left(x P^{\mu}+q^{\mu}\right)=x^{2} \mathcal{E}^{2} .
$$

For $u, d, s$ quarks we use, respectively,

$$
T_{u}=\frac{2}{3} T_{1}-T_{3}, \quad T_{d}=-\frac{1}{3} T_{1}-T_{3}, \quad T_{s}=-\frac{1}{3} T_{1},
$$

to get the unpolarised proton spin structure function averaging over three colours [19] $\left(f_{s}(x) \equiv 0\right.$ for $R$ in $\left.(19)\right)$

$$
g_{1}^{p}(x)=\frac{1}{2}\left[e_{u}^{2} \frac{1}{3} f_{u}(x)+e_{d}^{2} \frac{1}{3} f_{d}(x)\right] .
$$




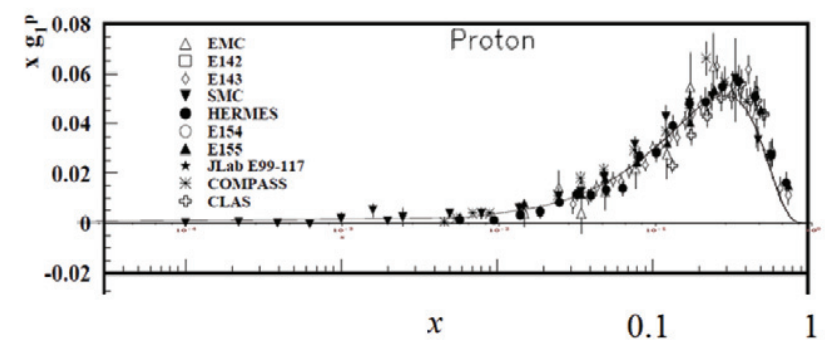

Fig. 2: Spin structure function of the proton (solid) from an exemplar calculation (24) based on flavour quarks derived from an intrinsic $U(3)$ configuration overlaid on experimental data [12] spanning four orders of magnitude. Figure taken from [8].

This represents rather well the experimentally extracted values [12] in fig. 2 without fitting parameters.

From the constituent quark model, we have an expression for the proton magnetic moment [23]

$$
\mu_{p}=\frac{4}{3} \mu_{u}-\frac{1}{3} \mu_{d}, \quad \mu_{q}=\frac{e_{q} \hbar / 2}{4 \pi \varepsilon_{0} m_{q}} .
$$

We use [19]

$$
m_{p}=2 m_{u}+m_{d}, \quad \frac{m_{u}}{m_{d}}=\frac{\int_{0}^{1} x f_{u}(x) \mathrm{d} x}{\int_{0}^{1} x f_{d}(x) \mathrm{d} x}=\frac{0.2722}{0.1432}
$$

and get $m_{u} c^{2}=371 \mathrm{MeV}, m_{d} c^{2}=195 \mathrm{MeV}$ from which

$$
\mu_{p}=2.779 \ldots \mu_{N}, \quad \mu_{N}=\frac{e \hbar / 2}{4 \pi \varepsilon_{0} m_{p}},
$$

to compare with experiment $2.7928473446(8) \mu_{N}[1]$.

Basic building blocks for QCD construction. -

We require local gauge invariance of a field Hamiltonian [24] constructed from the colour quark fields generated in $(16)^{2}$

$H=\int \psi^{\dagger}\left(-i \hbar c \boldsymbol{\alpha} \cdot \nabla+\beta m c^{2}\right) \psi \mathrm{d} x^{3}, \quad \psi^{\dagger}=\left(\psi_{1}^{*}, \psi_{2}^{*}, \psi_{3}^{*}\right)$.

Here we suppressed spinor indices which are mixed by the $4 \times 4$ Dirac matrices $\boldsymbol{\alpha}=\left(\alpha_{1}, \alpha_{2}, \alpha_{3}\right)$ and $\beta$. The spinor indices commute with the colour indices. Using left invariance of the coordinate fields $\left.\partial_{j}\right|_{u}=u i T_{j}=\left.u \partial_{j}\right|_{e}$ from (12) we get in the mass term of $(28)^{3}$

$$
\begin{aligned}
& \psi^{\prime}\left(u^{\prime}\right)^{\dagger} \psi^{\prime}\left(u^{\prime}\right)=\left(u^{\prime} i T_{j}[R]\right)^{\dagger}\left(u^{\prime} i T_{j}[R]\right)= \\
& \left(i T_{j}[R]\right)^{\dagger}\left(u^{\prime}\right)^{\dagger} u^{\prime}\left(i T_{j}[R]\right)=\psi(u)^{\dagger} \psi(u),
\end{aligned}
$$

\footnotetext{
${ }^{2}$ This section is edited from [25].

${ }^{3}$ Quark masses are ad hoc. One may speculate that the finite values of current masses $m_{u}, m_{d}$ represent a topological tension from mapping the curved intrinsic group to the flat algebra in laboratory space, somewhat like our interpretation of the electron as a peel-off from the neutron in the $n \rightarrow p$ decay [9] which scales (1) by $\pi a \equiv r_{e}$ via the strength of the electric charge in the classical electron radius, $m_{e} c^{2}=e^{2} /\left(4 \pi \varepsilon_{0} r_{e}\right)=\alpha \frac{\hbar c}{r_{e}}$. With flavour distributed over three colour charges, by analogy, $m_{q} c^{2}=\left(g_{s} / 3\right)^{2} /(4 \pi) \frac{\hbar c}{r_{q}}=\frac{\alpha_{s}}{9} \frac{\hbar c}{r_{q}}$. An interpretation of $1 / r_{q}$ as a curvature is supported by treating the $u, d, s$ quark mass matrix $M$ as an external field, cf. sect. 59 in [1]
}

provided the configuration variables $u^{\prime}, u$ are unitary, i.e., $\left(u^{\prime}\right)^{\dagger} u^{\prime}=u^{\dagger} u=1$. Next we impose the local gauge transformation

$$
\psi \rightarrow \psi^{\prime}=g(x) \psi, \quad g(x) \in S U(3), \quad \partial_{\mu} \rightarrow D_{\mu}=\partial_{\mu}+\mathcal{G}_{\mu},
$$

with colour gauge field, $\mathcal{G}=i g_{s} \mathbf{G}$ [1] containing a strong coupling $g_{s}$

$$
\mathcal{G}_{\mu}=i g_{s} G_{\mu}^{k} t_{k}, \quad k=1,2, \ldots, 8, \quad G^{k} \sim G^{(k)}(e)=\mathrm{d} \Phi_{e}\left(i t_{k}\right)
$$

and transforming (when $e \rightarrow g(x)$ in $G^{(k)}$ ) like in [27]

$$
\begin{aligned}
& \mathcal{G}_{\mu}^{\prime}=g(x) \mathcal{G}_{\mu} g(x)^{-1}-\partial_{\mu}(g(x)) g(x)^{-1} \rightarrow \\
& \left(D_{\mu}^{\prime} \psi^{\prime}\right)^{2}=\left(D_{\mu} \psi\right)^{2} .
\end{aligned}
$$

We thus have the basic ingredient colour fields for setting up QCD. Spin degrees of freedom enter from (10) and flavours enter from (11) and are extracted by (23). Choosing $u=g(x)$ in (30) and in (12) equates local gauge transformation in laboratory space to left translation of the intrinsic coordinate fields

$$
\psi_{j}(u)=\left.\partial_{j}\right|_{u}[\Phi]=\left.u \partial_{j}\right|_{e}[\Phi]=u \psi_{j}(e)
$$

Unflavoured baryons: $N$ and $\Delta$ states. - In (1) we multiply by $J$ and integrate over the off-toroidal degrees of freedom to get [9]

$$
\left[-\frac{1}{2} \sum_{j=1}^{3} \frac{\partial^{2}}{\partial \theta_{j}^{2}}+W(\boldsymbol{\theta})\right] R(\boldsymbol{\theta})=E R(\boldsymbol{\theta}), \quad \boldsymbol{\theta}=\left(\theta_{1}, \theta_{2}, \theta_{3}\right)
$$

for the dimensionless eigenvalues $E=\mathcal{E}_{0} / \Lambda$. The total potential

$$
W(\boldsymbol{\theta})=-1+\sum_{1 \leq i<j \leq 3}^{3} \frac{4 / 3}{16 \sin ^{2} \frac{1}{2}\left(\theta_{i}-\theta_{j}\right)}+\sum_{j=1}^{3} w\left(\theta_{j}\right)
$$

is periodic with $w$ from (4). The constant term -1 follows from differentiating through $J$ in the Laplacian (2) [15]; Dowker calls it the constant global curvature potential [28]. The centrifugal term has the numerator $4 / 3$ from the minimum value $\left(\mathbf{S}^{2}+\mathbf{M}^{2}\right) / \hbar^{2}=4[19]$ common for $N$ and $\Delta$ in integrating the second term in (2) over the six off-toroidal degrees of freedom contained in $\Upsilon$ from (15). Here the arbitrary labelling of the three eigenangles is exploited to make an average over the noncommuting components of $\mathbf{S}$ and $\mathbf{M}$ in (2). For a Wilson

quite analogous to the relation in general relativity between the curvature of the metric field and the energy-momentum density in spacetime. In stead of (8), we may embed the $2 D$ tori generated by $T_{u, d}$ from (23) in laboratory space to have $(x, y, z)=(a(1+$ $\left.\left.\cos e_{q} \theta_{1}\right) \cos \left(-\theta_{3}\right), a\left(1+\cos e_{q} \theta_{1}\right) \sin \left(-\theta_{3}\right), a \sin e_{q} \theta_{1}\right)$. Using [26] we find Gaussian curvatures $K=\frac{\cos e_{q} \theta_{1}}{a e_{q}\left(a+a \cos e_{q} \theta_{1}\right)}$ with $e_{q}$ in units of $e$. Integration with $R^{2}$ from (19) over $[0, \pi]^{3}$ yields $\frac{1 / r_{d}}{1 / r_{u}}=\sqrt{\frac{\left|\left\langle K_{d}\right\rangle\right|}{\left|\left\langle K_{u}\right\rangle\right|}}=$ 2.19 to compare with $m_{d} / m_{u}=2.16(40)[1]$. 
analogue [29], which is more commonly used, $w$ would read $w_{\text {Wilson }}(\theta)=(1-\cos \theta)$. However, the Wilson analogue does not represent well even the lowest-lying states if used in (34) in stead of the Manton-inspired potential we use here.

The total potential $W$ has periodicity $2 \pi$ in all three eigenangles. This opens for the introduction of Bloch wave degrees of freedom. The measure-scaled toroidal wave function $R$ is antisymmetric under interchange of the three colour degrees of freedom $\theta_{j}$ and we may therefore expand it on Slater determinants [19] (see Supplementary Material Supp1_1Deigenvalues_Matlab.m and Supp1a_1Deigenvalues_Matlab.pdf (SM1), Supp21DbaseFunctionSet-Maple.mw and Supp2a-1Dbase FunctionSet-Maple.pdf (SM2), Supp3-3DchargedNMathcad.mcd and Supp3a-3DchargedN-Mathcad (SM3))

$$
g_{l m n}=\left|\begin{array}{ccc}
\phi_{l}\left(\theta_{1}\right) & \phi_{l}\left(\theta_{2}\right) & \phi_{l}\left(\theta_{3}\right) \\
\phi_{m}\left(\theta_{1}\right) & \phi_{m}\left(\theta_{2}\right) & \phi_{m}\left(\theta_{3}\right) \\
\phi_{n}\left(\theta_{1}\right) & \phi_{n}\left(\theta_{2}\right) & \phi_{n}\left(\theta_{3}\right)
\end{array}\right|, \quad l, m, n \in \mathbb{Z}^{+},
$$

constructed from solutions $\phi_{i}$ with periodicity either $2 \pi$ or $4 \pi$ to the one-dimensional problem

$$
\left[-\frac{1}{2} \frac{\mathrm{d}^{2}}{\mathrm{~d} \theta^{2}}+w(\theta)\right] \phi_{i}(\theta)=e_{i} \phi_{i}(\theta) .
$$

These correspond to Bloch wave functions [30]

$$
\phi(\theta)=e^{i \kappa \theta} b(\theta),
$$

with $b$ carrying the $2 \pi$ periodicity of the potential and $\kappa=0, \kappa= \pm \frac{1}{2}$, respectively. No other values of $\kappa$ are allowed since the square of the wave function must be single-valued on the configuration space to keep up its probability interpretation. Note that in (37) there is no summation over $i$. Table 1 shows the first eigenvalues $e_{i}$ for real-valued wave functions emulating Bloch wave vectors $\kappa=0$ (2 $2 \pi$ periodicity) and $e_{i}^{\prime}$ for $\kappa= \pm \frac{1}{2}$ ( $4 \pi$ periodicity). The eigenvalues are found by collocation (see SM1). We get an eigenvalue $E=e_{l}+e_{m}+e_{n}$ for (36) under an approximate, separable Hamiltonian ${ }^{4}$

$$
\left[\sum_{j=1}^{3}\left(-\frac{1}{2} \frac{\partial^{2}}{\partial \theta_{j}^{2}}+w\left(\theta_{j}\right)\right)\right] g_{l m n}=E g_{l m n} .
$$

Combining three different eigenvalues from table 1 gives the resonance structure shown in fig. 3 for maximally charged states, i.e., $N^{+}$-like and $\Delta^{++}$-like states. We note in passing the resulting value for the relative neutron-to-proton mass shift [31],

$$
\begin{aligned}
& \frac{m_{n}-m_{p}}{m_{p}} \approx \\
& \frac{e_{1}+e_{2}+e_{3}-\left(e_{1}^{\prime}+e_{2}^{\prime}+e_{3}\right)}{e_{1}^{\prime}+e_{2}^{\prime}+e_{3}}=0.13847(14) \%,
\end{aligned}
$$

${ }^{4}$ The eigenvalues of this Hamiltonian are closer to those involving the full potential (35) than one might expect. This is because the total curvature term and the centrifugal terms have opposite signs and more or less cancel each other when integrated [31].
Table 1: 1D eigenvalues (37) to construct the approximate

\begin{tabular}{|c|c|c|c|}
\hline $\begin{array}{c}i \\
\text { Level }\end{array}$ & $\begin{array}{c}e_{i} \\
\text { Eigenvalue }\end{array}$ & $\begin{array}{c}e_{i}^{\prime} \\
\text { Diminished }\end{array}$ & $\begin{array}{c}e_{i}^{\prime} \\
\text { Augmented }\end{array}$ \\
\hline 1 & 0.499804708 & & 0.5001727904 \\
\hline 2 & 1.502988968 & 1.496433950 & \\
\hline 3 & 2.471378779 & & 2.522629649 \\
\hline 4 & 3.600509000 & 3.377236032 & \\
\hline 5 & 4.218515963 & & 4.803947527 \\
\hline$\cdots$ & $\cdots$ & $\cdots$ & $\ldots$ \\
\hline
\end{tabular}
baryon spectrum in fig. 3 from (36). The eigenvalues are calculated with 1500 collocation points (sm1). The lowest eigenvalues, as expected, are close to those of the ordinary harmonic oscillator. Moving up to higher levels, the eigenvalues increase quadratically as indicated in fig. 4. Table extract from [19].

to compare with the observed value $0.13784190(5) \%$ [1]. Note that we combine two period doublings in the choice of $\kappa$ 's for the protonic state. The choices are shown with black dots in fig. 4. The same kind of coupling between Bloch wave vectors is necessary when the full potential $W$ in (35) is to be used for exact solutions of (1) since then the singularity of the centrifugal term can be cancelled. Unfortunately we have not succeeded in finding a complete base on which to expand protonic states for solving with analytical integrals. Our best suggestion so far is $[19,25]$

$$
\begin{aligned}
& f_{p q r}=\left|\begin{array}{ccc}
\cos p \theta_{1} & \cos p \theta_{2} & \cos p \theta_{3} \\
\sin q \theta_{1} & \sin q \theta_{2} & \sin q \theta_{3} \\
\cos r \theta_{1} & \cos r \theta_{2} & \cos r \theta_{3}
\end{array}\right| \in R_{n} \quad \text { and } \\
& \frac{1}{2}\left(e^{\frac{i}{2}\left(\theta_{1}+\theta_{2}+\theta_{3}\right)}+e^{\frac{-i}{2}\left(\theta_{1}+\theta_{2}+\theta_{3}\right)}\right) f_{p q r} \in R_{p}
\end{aligned}
$$

with non-negative integers $0 \leq p<r$ and $0<q$. For $R_{n}$ the set is complete and yields the very accurate neutron-to-electron mass ratio $[9,32]$ (see Supplementary Material Supp4-3DneutralN-Mathcad.mcd and Supp4a-3DneutralN-Mathcad.pdf (SM4))

$$
\frac{m_{n}}{m_{e}}=\frac{\pi}{\alpha\left(m_{n}\right)} E_{n}=1839(1), \quad E_{n}=4.382(2),
$$

in agreement with the observed value [1]. However, for the protonic state $R_{p}$ we have not settled how to select the $p, q, r$-triples to keep completeness and avoid overcompleteness. An abstract crystallographic approach is needed; Jones [33] seems appropriate.

Charge singlet states: neutral pentaquarks? A particularly interesting set of states with no charged partners and parity opposite to those from (41) are

$$
f_{p q r}^{0}=\left|\begin{array}{ccc}
\cos p \theta_{1} & \cos p \theta_{2} & \cos p \theta_{3} \\
\cos q \theta_{1} & \cos q \theta_{2} & \cos q \theta_{3} \\
\cos r \theta_{1} & \cos r \theta_{2} & \cos r \theta_{3}
\end{array}\right|, \quad 0 \leq p<q<r \in \mathbb{N}
$$




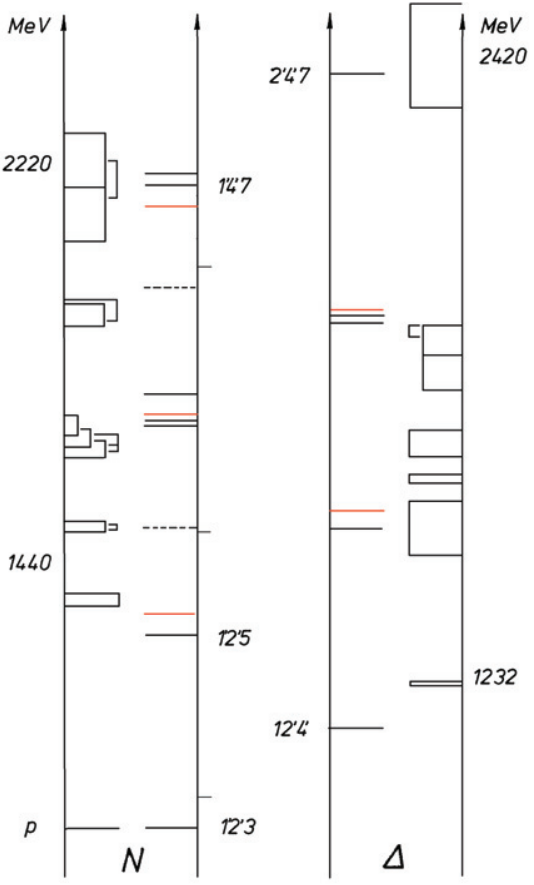

Fig. 3: Unflavoured baryon spectra. The dashed lines are singlet states. The red lines mark states with augmented contribution in level 3. All lines are approximate predictions based on (39). The boxes represent baryons observed with certainty [1]. The box widths represent the uncertainty in the mass pole peaks, not resonance widths, which are much larger. Figure updated from [31].

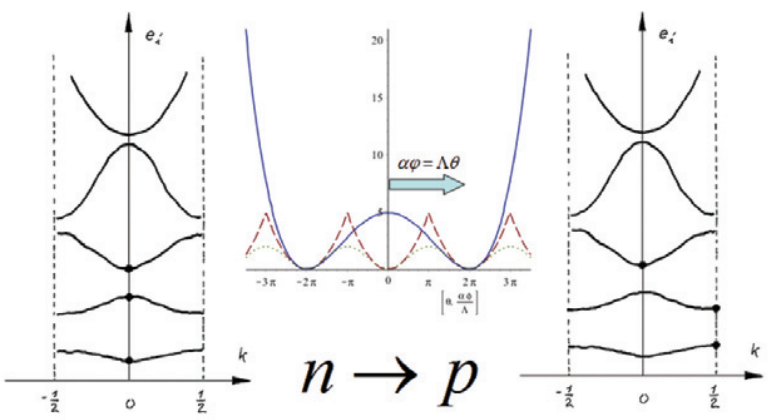

Fig. 4: Left and right: reduced zone schemes, cf. [30], for Bloch wave numbers for the neutron state (left) and the proton state (right). Middle: Higgs potential (solid, blue) matching the Manton-inspired potential [14] (dashed, red) and the Wilson-inspired potential [29] (dotted, green). The Manton and Wilson inspired potentials yield the same value for the Higgs mass and the electroweak energy scale [19] whereas only the Manton inspired potential (6) gives a satisfactory reproduction of the baryon spectrum seen in fig. 3. Figure adapted from [34].

Like for the neutral states $R_{n}$ in (41) also the Hamiltonian in (1) for (43) can be diagonalized with a Rayleigh-Ritz method $[19,32]$ where the integrals needed for the Hamiltonian matrix elements can be found analytically and the
Table 2: Scarce singlet states. Eigenvalues based on Slater determinants (43) of three cosines up to order 20 (see SM5). The first column shows eigenvalues from the approximate Hamiltonian (39) and the third column shows eigenvalues of the exact equation (34). The rest masses are predicted from a common fit of the neutron state $939.57 \mathrm{MeV}$ to the ground state 4.382 of (34) with no period doublings. The four resonances marked by an asterix $(*)$ lie within the observational window in $\Lambda_{b}^{0}$ decays (44) at LHCb. Table updated from [31].

\begin{tabular}{cccc}
\hline $\begin{array}{c}\text { Singlet } \\
\text { approximate }(39)\end{array}$ & $\begin{array}{c}\text { Toroidal } \\
\text { label }\end{array}$ & $\begin{array}{c}\text { Singlet } \\
\text { exact }(34)\end{array}$ & $\begin{array}{c}\text { Rest mass } \\
\mathrm{MeV} / c^{2}\end{array}$ \\
\hline 7.1895 & 135 & 7.1217 & 1527 \\
$\ldots$ & $\ldots$ & $\ldots$ & $\ldots$ \\
18.9214 & 1511 & 19.7327 & 4231 \\
20.3774 & 579 & 20.9940 & $4501^{*}$ \\
20.8910 & 3511 & 21.7110 & $4655^{*}$ \\
21.0766 & 1711 & 22.0409 & $4726^{*}$ \\
23.0609 & 3711 & 23.7887 & $5101^{*}$ \\
24.4575 & 1911 & 23.9981 & 5146 \\
$\ldots$ & $\ldots$ & $\ldots$ & $\ldots$ \\
\hline
\end{tabular}

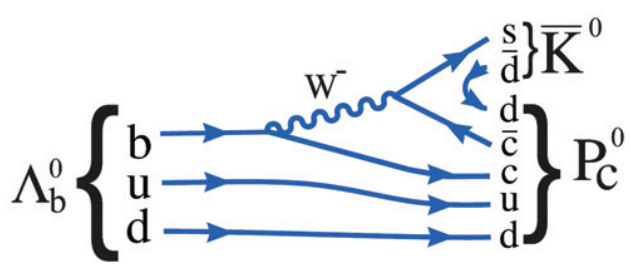

Fig. 5: Feynman diagram for neutral pentaquark formation (44). Figure adapted from [35].

eigenvalues therefore be found with high accuracy. The eigenvalues for these cases are given in table 2 (see Supplementary Material Supp5-3Dsinglets-Mathcad.mcd and Supp5a-3Dsinglets-Mathcad.pdf (SM5)).

We have previously suggested to look for neutral charge resonances in [31] and had the opportunity to discuss the possibilities at LHCb with Sheldon S. Stone at the EPSHEP 2015 after Marta Calvi had been so kind as to forward our request. Sheldon Stone suggested the following channel (when enough data are acquired) [36]:

$\Lambda_{b}^{0} \rightarrow \bar{K}^{0}+P_{c}^{0} \rightarrow \bar{K}^{0}+J / \Psi+\Delta^{0} \rightarrow \bar{K}^{0}+J / \psi+p+\pi^{-}$.

Figure 5 shows a quark structure interpretation for $P_{c}^{0}$ production in $\Lambda_{b}^{0}$ decay which can be reached at $\mathrm{LHCb}$. Other channels could be narrow resonances in photoproduction on neutrons, in $\pi^{-} p$ scattering and in invariant mass spectra of $\Sigma_{c}^{+}(2455) D^{-}$from decays.

Conclusion. - We derived quark and gluon fields for QCD from baryonic states on an intrinsic $U(3)$ Lie group configuration space with a mass Hamiltonian. We have shown in general that the intrinsic variable must be unitary for the mass term of the field Hamiltonian to be 
invariant under translations in the intrinsic space and that local gauge transformations in laboratory space correspond to left translations in configuration space. As applications of the momentum form, we derived a proton spin structure function and a proton magnetic moment. We used the conjugacy of coordinate fields and coordinate forms as a generalisation of action-angle quantisation in ordinary quantum mechanics. We hinted at a topological origin of quark masses which should be better understood.

We have shown how to derive unflavoured baryon spectra without explicit introduction of quarks and gluons. Colour degrees of freedom are carried by the toroidal degrees of freedom and spin and flavour by off-toroidal derivatives in the Laplacian on $U(3)$. The unflavoured spectrum from approximate calculations compares well with observed four star resonances. We have given an approximate value for the relative neutron to proton mass shift and an accurate value for the neutron mass. We predict unflavoured neutral charge singlets that might be interpreted as neutral pentaquarks.

$$
* * *
$$

I thank for the referee queries to clarify the physical insight intended. I thank EPS-HEP2019 and Alps 2020 for accepting my work for presentation. Alas, corona cancelled the latter.

\section{REFERENCES}

[1] Particle Data Group (Zyla P. A. et al.), Prog. Theor. Exp. Phys., 2020 (2020) 083C01.

[2] Isgur N. and Karl G., Phys. Rev. D, 18 (1978) 4187.

[3] Gell-Mann M., Discussion after Plenary Session VI, Strange particle physics. Strong Interactions II, Rapporteur SNow G. A., in Proceedings of the International Conference on High-Energy Nuclear Physics, Geneva, 1962 (CERN Scientific Information Service, Geneva, Switzerland) 1962, p. 805.

[4] Uhlenbeck G. E. and Goudsmit S. A., Naturwissenschaften, 13 (1925) 953.

[5] Schiff L. I., Quantum Mechanics, 3rd edition (McGrawHill, Kogakusha) 1968, p. 209.

[6] Duncan A., The Conceptual Framework of Quantum Field Theory (Oxford University Press, Oxford, UK) 2012, p. 181.

[7] Trinhammer O. L., EPL, 125 (2019) 41001.

[8] Trinhammer O. L., EPL, 124 (2018) 31001.

[9] Trinhammer O. L., EPL, $102 \quad$ (2013) 42002 (arXiv:1303.5283v2 [physics.gen-ph] (22 April 2014)).

[10] Heisenberg W., Ann. Phys., 32 (1938) 20.

[11] Landau L. D. and Lifshitz E. M., The Classical Theory of Fields, Course of Theoretical Physics, Vol. 2, 4th edition (Elsevier Butterworth-Heinemann, Oxford) 2005, p. 97.
[12] Particle Data Group (Tanabashi M. et al.), Phys. Rev. D, 98 (2018) 030001.

[13] Kogut J. B. and Susskind L., Phys. Rev. D, 11 (1975) 395.

[14] Manton N. S., Phys. Lett. B, 96 (1980) 328.

[15] Trinhammer O. L. and Olafsson G., The Full LaplaceBeltrami operator on $U(N)$ and $S U(N)$, arXiv:mathph/9901002v2 (10 April 2012).

[16] Weyl H., The Classical Groups. Their Invariants and Representations, Princeton Landmarks in Mathematics (Princeton University Press, NJ) 1966, p. 197.

[17] Milnor J., Ann. Math. Stud., 51 (1963) 1.

[18] Merzbacher E., Quantum mechanics (John Wiley and Sons, Inc., New York, London) 1961, p. 187.

[19] Trinhammer O. L., Intrinsic Quantum Mechanics Behind the Standard Model. From Lie Group Configurations to Particle Physics Observations, Research Gate, December 2018 (book), p. 85 , p. 90 , p. 107 , p. 43 , p. 102 , p. 162.

[20] Warner F. W., Foundations of Differentiable Manifolds and Lie Groups (Springer, New York) 1983, p. 105.

[21] Bohr A. and Mottelson B. R., Nuclear Structure, Vol. 1 (W. A. Benjamin, New York, Amsterdam) 1969, p. 87 .

[22] Trinhammer O. L., EPL, 128 (2019) 11004.

[23] Donoghue J. F., Golowich E. and Holstein B. R., Dynamics of the Standard Model, 2nd edition (Cambridge University Press, Cambridge) 2014, p. 339.

[24] Sakurai J. J., Advanced Quantum Mechanics (AddisonWesley, Redwood City) 1967, p. 145.

[25] Trinhammer O. L., PoS(EPS-HEP2019) (2019) 616 (arXiv:2007.02936 [hep-ph] (6 July 2020)).

[26] Irons M. L., The Curvature and Geodesics of the Torus (2005), http://www2.rdrop.com/ half/math/torus/ torus . curvature.pdf.

[27] SCHECK F., Electroweak and Strong Interactions. Phenomenology, Concepts, Models, 3rd edition (Springer, Berlin) 2012, p. 223.

[28] Dowker J. S., Ann. Phys., 62 (1971) 361.

[29] Wilson K. G., Phys. Rev. D, 10 (1974) 2445.

[30] Ashcroft N. W. and Mermin N. D., Solid State Physics (Holt, Reinhart and Winston, New York) 1976, p. $133,160$.

[31] Trinhammer O. L., Neutron to proton mass difference, parton distribution functions and baryon resonances from dynamics on the Lie group u(3), arXiv:1109.4732v3 [hepth] (25 June 2012).

[32] Trinhammer O. L., EPL, 131 (2020) 31001.

[33] Jones H., The Theory of Brillouin Zones and the Electronic States in Crystals, (North-Holland Publishing Company, Amsterdam) 1960, p. 112.

[34] Trinhammer O. L., Bohr H. G. and Jensen M. S., PoS(EPS-HEP2015) (2015) 097.

[35] LHCb Collaboration (Aaij R. et al.), Phys. Rev. Lett., 115 (2015) 072001 (arXiv:1507.03414v2 [hep-ex] (20 July 2015)).

[36] Stone Sheldon S., private communications, Syracuse University, USA, 2016 and 2019. 